\title{
The Doctor's PDA and Smartphone Handbook Personal digital assistant
}

\author{
Mohammad Al-Ubaydli ${ }^{1} \quad$ Chris Paton ${ }^{2}$
}

J R Soc Med 2005;98:494-495

This is the first of a series of extracts from a forthcoming book by A. Al-Ubaydli and $C$ Paton

[www.rsmpress.co.uk/bkpda.htm].

\section{INTRODUCTION}

What do drug dealers and doctors have in common? From the 1980s onwards neither could do their job without a pager. Only by carrying a pager can junior doctors leave their wards, safe in the knowledge that they would be paged about their patients' needs. The same junior doctors also feel safer knowing that they can page their senior at any time to get advice and support. And of course the code blue message on pagers is essential to the ability of the cardiac arrest team to respond quickly wherever its individual members are dispersed in the hospital.

Handheld computers promise an even bigger qualitative contribution to clinical workflow. Not only can you use a handheld computer to do your clinical work faster and better than before, you can do some things that colleagues without these devices are simply incapable of doing.

\section{WHAT IS A HANDHELD COMPUTER?}

A handheld computer is a computer small enough to hold in your hand or fit into your coat pocket. It is often called a 'personal digital assistant' (PDA). Some handheld computers also have phone features, enabling the owner to make and receive phone calls - they are called smartphones.

Of all the computer devices, handheld computers are possibly the most appropriate for clinical practice. While working with IT departments around the UK and USA we heard the same comment from puzzled computer professionals: it was so hard to convince clinicians to adopt computers. However, this changed with the arrival of handheld computers. Doctors are regularly buying them in order to improve patient care-even before their IT departments decided to make the investment. Healthcare computing professionals around the world are delighted that the clinicians now care passionately about computing resources because of PDAs and smartphones.

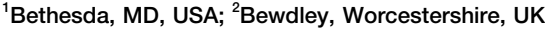

Correspondence to: Dr Mohammad Al-Ubaydli

E-mail: me@mo.md
There are several aspects of handheld computers that make them well-suited to clinical practice.

First, of course, is the portability. The devices are small enough to carry everywhere, including from ward rounds to patients' homes, and lecture to libraries.

Battery life makes the portability qualitatively different from laptops or tablet PCs. Most handheld computers are usable over 2 days of clinical work - few laptops or tablet PCs can last 3 hours without requiring recharging. The efficiency of the battery still supports speed. Laptops and tablet PCs preserve battery life by switching to 'standby' or 'hibernation' modes after a few minutes without use. The former means the computer will take several seconds to respond, while up to a minute is necessary to use a computer that is in hibernation mode. By contrast, a handheld computer instantly works no matter how long it has been since you last used it. This is necessary for the continuous interruption environment of ward rounds.

Every handheld computer includes a cradle for recharging. The former sits in the latter for a couple of hours to recharge completely. The cradle also allows synchronization.

Synchronization is the process of copying everything you have on your handheld computer onto your PC. This means that anything you do on your handheld computer is backed up on your PC the next time you synchronize. Compare this with backing up a paper diary.

Synchronization is a two-way process. If you type a new appointment into your PC's diary the new appointment will appear on your handheld computer the next time you synchronize. And if you write down the details of a test for one of your patients on your handheld computer the details will appear on your PC the next time you synchronize. This is great for sharing information that you write on your handheld computer and your secretary types on your PC.

Beaming is another way to share information. Line up one handheld computer with another then beam information between the two. This means you can send details about your patients to a colleague's device while the two of you discuss the most important points - or simply enjoy your tea.

Handheld computers allow writing. Some can recognize handwriting some of the time, and this is 
a tremendous achievement given the handwriting of doctors. But the fastest and most reliable way to write is using a slightly modified alphabet without joining up the letters. This may not be as fast as your writing on paper, but it will be legible and more than one doctor has noticed an improvement in their writing on paper after using a handheld computer.

The advantage of writing rather than typing is that you can enter information into your handheld computer during the ward round. For prolonged writing you may prefer to use an unfolding keyboard instead. Unfolded this is the same size and comfort of a normal PC allowing fast typing. And folded it is the same size as your other coat pocket, maintaining portability (and perhaps adding some balance to your gait).

You can enter all sorts of information in your handheld computer. The original devices were named PDAs because of the included organizer software: diary, address book, task list and simple notes. A good rule of thumb is that if you find yourself needing a piece of information more than once then you should take the trouble to enter it into your handheld computer. The date of an upcoming lecture, the phone number of your primary care trust manager, the tasks you must carry out for your patients today and notes from the lecture you attended yesterday will all be useful to refer to in the future.

Extra software allows entry of other information. For example, databases allow surgeons to keep logbooks and general practitioners to maintain their personal development plans. Reference software provides access to the British National Formulary as well as American and German formularies. You can read textbooks from around the world without the burden of bookshelves. And with the support of your hospital's computer department doctors on the ward can read X-ray reports as soon as the radiologist types them and blood test results as soon as the pathology laboratory produces them.

In fact, computer departments of healthcare institutions around the world are making such investments.

\section{HANDHELD COMPUTER USE AROUND THE WORLD}

PubMed lists almost 400 papers from 2003 and 2004 that covered handheld computers and the rate for 2005 is probably accelerating. [The medical subject heading 'computers, handheld' is useful for finding these.] More significantly, the character of the papers is changing from anecdotal cases and reviews to quantitative trials and sophisticated projects.

At Stanford University, for example, students were provided with handheld computers and teachers used them in tutorials. The teachers would periodically ask questions and the students would select their answers on the handheld computers. The students' aggregated choices would appear on the teacher's computer. The anonymity gave the students the confidence to give answers based on their understanding of the topic - the results gave the instructors instant feedback about the class's progress.

Such work is the reason for medical schools of Harvard University, the University of Cambridge and the National University of Singapore providing handheld computers to their students.

Clinical work has also benefited. At St John's Hospital in Scotland doctors on ward rounds have access to each patient's admission details, pathology laboratory test results and notes from previous ward rounds, as well as reference documents. Meanwhile in Lanarkshire the night-time hospital emergency care team of two nurses and five doctors uses handheld computers for triaging all patients, structured clinical assessments, prescriptions and protocols. The devices generate printed clinical assessments at the bedside and hand-over registers and reports for the morning teams.

It is such work that convinced Duke University Health System's hospitals in the USA, St Olavs Hospital in Norway and Shin-Kong Wu Ho-Su Memorial Hospital in Taiwan to invest in handheld computers to support patient care. Even without expensive integrated electronic systems the devices have shown a return on the investment. This is why Satellife has deployed simple battery-powered handheld computers in Uganda to collect public health data.

\section{Clinical vignette of a Medical Digital Assistant}

In doing locum work around the UK's hospitals Dr ibn Battuta always used his handheld computer. With it he kept track of the phone numbers of his consultant and his locum agency, the shifts he was to do, the jobs for each of the patients under his care, and the directions to the different hospitals he was to practise in. On ward rounds of different specialties he referred to the British National Formulary and the 20 specialist textbooks he carried with him at all times.

Finally, for revalidation, he kept track of the lessons he learnt every day. At the end of the year he had ample proof that he was keeping up to date with the literature and improving his clinical skills.

In many of the hospitals he found colleagues who also used the devices. Those members of his team would beam him more phone numbers from the hospital directory. At the end of his shift he would beam to them jobs for the patients that had not yet been completed. He could trust that the information would be transferred accurately and comprehensively, as well as documenting the jobs that he had already completed.

In a few hospitals he was delighted to see institutional support for the devices. The IT departments had deployed software around the hospital to ensure that patient information would never have to be entered more than once. The time saved was considerable: on ward rounds his team would have access to each patient's demographic details, investigation results and previous notes on their handheld computer. But there was improved patient safety because dangerous blood results were clearly highlighted and prescription errors were reduced by warnings from the software.

At one hospital he was even provided with his own smartphone on the first day. The nurses would send text messages or directly phone the devices, the operator explained, because it was much faster than paging and waiting for the doctors to reply. Dr ibn Battuta was glad to see that others were realising the advantages that he treasured in his own device. 